\title{
Teores foliares de NPK em gravioleira sob adubação orgânica e mineral
}

\author{
Foliar NPK in soursop under organic and mineral fertilization
}

\author{
Altamiro Oliveira de Malta 1 \\ Msc. em Agronomia, Universidade Federal da Paraíba, Centro de Ciências Agrárias, Campus II \\ E-mail: altamiro1@ig.com.br
}

Walter Esfrain Pereira 2

Prof. Dr. do Departamento de Ciências Fundamentais e Sociais, Universidade Federal da Paraíba, Centro de

Ciências Agrárias, Campus II

E-mail: walterufpb@yahoo.com.br

\begin{abstract}
Mariana Neves Nóbrega Torres 3
Graduada em Agronomia, Universidade Federal da Paraíba, Centro de Ciências Agrárias, Campus II

E-mail: marianannobrega@gmail.com
\end{abstract}

\begin{abstract}
Alan Oliveira de Malta 4
Graduando em Agronomia, Universidade Federal da Paraíba, Centro de Ciências Agrárias, Campus II

E-mail: alan-oliveira@live.com
\end{abstract}

Diego Almeida Medeiros 5
Msc. em Agronomia, Universidade Federal da Paraíba, Centro de Ciências Agrárias, Campus II
E-mail: diegoa083@gmail.com

Jefferson Alves Dias 6

Msc. em Agronomia, Universidade Federal da Paraíba, Centro de Ciências Agrárias, Campus II

E-mail: jefferson_cz@hotmail.com

Resumo: Nos últimos anos, a fruticultura tornou-se um promissor setor de investimento. A gravioleira é uma frutífera cultivada em todo o Brasil, sendo a região Nordeste a que possui as maiores áreas implantadas. No entanto, a produção dessa cultura ainda é considerada baixa devido principalmente à escassez de informações sobre as técnicas adequadas de manejo, incluindo a nutrição da planta. Desta forma, o objetivo deste experimento foi avaliar os teores foliares de NPK em gravioleira sob adubação orgânica e mineral. Foi instalado um experimento em um pomar de gravioleira com 42 meses de idade entre os meses de janeiro a setembro de 2014. O delineamento experimental foi em blocos casualizados, com sete tratamentos e quatro repetições, com duas plantas por parcela, no espaçamento de 5,0 $\mathrm{m} \times 5,0$ $\mathrm{m}$. Os tratamentos utilizados foram: $\mathrm{T}_{1}=$ testemunha (sem adubação); $\mathrm{T}_{2}=$ adubação mineral $(\mathrm{NPK}) ; \mathrm{T}_{3}=$ esterco de bovino; $\mathrm{T}_{4}=$ esterco de aves; $\mathrm{T}_{5}=$ adubação mineral + esterco de caprino; $\mathrm{T}_{6}=$ esterco de aves $+3 \mathrm{~kg}$ de pó de rocha; $\mathrm{T}_{7}=$ esterco de caprino. As variáveis analisadas foram: teores foliares de nitrogênio, fósforo e potássio. Os dados foram submetidos à análise de variância e ao teste de Tukey. A adubação orgânica com esterco bovino proporcionou maiores teores foliar de fósforo e potássio; O esterco caprino, seguido da adubação com NPK e esterco bovino promoveram maiores teores foliar de nitrogênio.

Palavras-chave: Annona muricata L.; Manejo da fertilidade do solo; Nutrição de plantas. 


\begin{abstract}
In recent years, fruit-growing has become a promising investment sector. The soursop is a fruit tree cultivated throughout Brazil, being the Northeast that has the largest areas implanted. However, the production of this crop is still considered low due mainly to the scarcity of information on the appropriate management techniques, including plant nutrition. Thus, the objective of this experiment was to evaluate NPK foliar contents in soursop under organic and mineral fertilization. An experiment was carried out in a 42 month-old soursop orchard from January to September 2014. The experimental design was a randomized complete block design with seven treatments and four replications, with two plants per plot, at a spacing of 5,0 $\mathrm{m} \times 5,0 \mathrm{~m}$. The treatments used were: $\mathrm{T} 1=$ control (without fertilization); $\mathrm{T} 2=$ mineral fertilization $(\mathrm{NPK}) ; \mathrm{T} 3=$ bovine manure; $\mathrm{T} 4=$ poultry manure; $\mathrm{T} 5$ $=$ mineral fertilization + goat manure; $\mathrm{T} 6=$ poultry manure $+3 \mathrm{~kg}$ of rock dust; $\mathrm{T} 7=$ goat manure. The analyzed variables were leaf, nitrogen, phosphorus and potassium contents. Data were submitted to analysis of variance and Tukey test. Organic fertilization with bovine manure provided higher leaf phosphorus and potassium contents; Goat manure, followed by fertilization with NPK and bovine manure promoted higher nitrogen contents.
\end{abstract}

Keywords: Annona muricata L.; Soil fertility management; Plant nutrition.

\title{
1 Introdução
}

A gravioleira (Annona muricata L.) é uma das importantes frutíferas cultivadas no Nordeste brasileiro, região onde se adapta muito bem, principalmente nos Estados da Paraíba, Ceará, Pernambuco e Bahia (SACRAMENTO et al., 2003; TELES et al., 2017), seus frutos são comercializadas sob a forma de fruta fresca, polpa fresca ou congelada, sendo ainda utilizadas no preparo de sucos, geleias, iogurtes, sorvetes, entre outras finalidades alimentares (SÃO JOSÉ et al., 2014).

Por ser uma espécie que se adapta aos mais variados fatores edafoclimáticos, em muitos pomares é negligenciada quanto ao manejo coreto das plantas, não se empregando adequadamente as tecnologias disponíveis (adubação, irrigação, controle de pragas, dentre outros), o que limita o potencial produtivo do pomar (FREITAS et al., 2013). Entretanto, há muitos pomares onde a produção é obtida sobe nível tecnológico, garantindo elevada produtividade (SÃO JOSÉ, 2003).

O manejo da adubação é uma prática que permite a reposição de nutrientes essências às plantas, deve considerar a dificuldade em se aliar a produtividade à qualidade do produto colhido, visto que o aspecto nutricional pode afetar características importantes do fruto como, cor, sabor, tamanho, dentre outras (MALAVOLTA, 1994), assim, o produtor pode se beneficiar da qualidade dos frutos, do estado fitossanitário e nutricional das plantas, bem como da produtividade de seu pomar (ABREU et al., 2005). 
De acordo com São José et al. (2014) em regiões produtoras de graviola, observa-se que plantas bem nutridas apresentam produção continuada ao longo do ano e que solos com adequada disponibilidade de nutrientes favorece o crescimento vegetativo e reprodutivo. Conforme Gomes Júnior et al. (2018) o K e N são os elementos mais requeridos pela gravioleira, com elevadas demandas desde a fase vegetativa até a frutificação.

Soares et al. (2007) avaliaram o crescimento e composição mineral de mudas de gravioleira em resposta a doses de fósforo, afirmaram que os nutrientes que mais acumularam na parte aérea seguem a seguinte ordem decrescente para os macronutrientes $\mathrm{N}, \mathrm{K}, \mathrm{Ca}, \mathrm{P}, \mathrm{S}$ e Mg. Souza et al. (2003), Holanda Filho et al. (2006) e Samarão et al. (2011) ressaltam a importância da adubação na nutrição e no crescimento da gravioleira.

Diante da importância da nutrição da planta e aos poucos trabalhos abordando a gravioleira, esse estudo teve como objetivo avaliar os teores foliares de NPK em gravioleira sob adubação orgânica e mineral.

\section{Metodologia}

O experimento foi conduzido em pomar de gravioleiras da cultivar 'Morada', pertencente à Fazenda Quaty III, distrito de Santa Maria, localizado no município de Areia, PB. O referido município encontra-se inserido na mesorregião do Agreste paraibano e microrregião do Brejo paraibano, com as coordenadas geográficas de $6^{\circ} 57^{\prime} 42^{\prime \prime} \mathrm{S}$ e $35^{\circ} 41^{\prime}$ 43" W, a $618 \mathrm{~m}$ de altitude (GONDIM, 1999). O solo da área experimental é classificado como Latossolo Amarelo Distrófico. A vegetação nativa é de floresta subperenifólia e o relevo é fortemente ondulado (BRASIL, 1972).

As gravioleiras no início da pesquisa estavam com 42 meses de idade. O experimento foi realizado entre os meses de janeiro a setembro de 2014. O delineamento experimental foi em blocos casualizados, com sete tratamentos e quatro repetições, com duas plantas por parcela, no espaçamento de 5,0 $\mathrm{m}$ x 5,0 $\mathrm{m}$. Sendo: $\mathrm{T}_{1}=$ testemunha (sem adubação); $\mathrm{T}_{2}=$ adubação química $(\mathrm{NPK}) ; \mathrm{T}_{3}=$ esterco de bovino; $\mathrm{T}_{4}=$ esterco de aves; $\mathrm{T}_{5}=\mathrm{NPK}+$ esterco de caprino, $\mathrm{T}_{6}=$ esterco de aves $+3 \mathrm{~kg}$ de pó de rocha e $\mathrm{T}_{7}=$ esterco de caprino.

A adubação mineral foi realizada de acordo com a análise química do solo, levando em consideração os macronutrientes ( $\mathrm{P}$ e K). Quanto ao nitrogênio, a recomendação foi de acordo com Cavalcanti (2008), para a cultura da gravioleira em produção. Os atributos químicos do solo, na camada 0-20 $\mathrm{cm}$ de profundidade, apresentou os seguintes valores: $\mathrm{pH}=$ 4,9; $\mathrm{P}=3,67 \mathrm{mg} \mathrm{dm}^{-3} ; \mathrm{K}^{+}=39,00 \mathrm{mg} \mathrm{dm}^{-3} ; \mathrm{Na}^{+}=0,07 \mathrm{cmol}_{\mathrm{c}} \mathrm{dm}^{-3} ; \mathrm{H}^{+}+\mathrm{Al}^{3+}=8,0 \mathrm{cmol}_{\mathrm{c}} \mathrm{dm}^{-}$ ${ }^{3} ; \mathrm{Al}^{3+}=0,85 \mathrm{cmol}_{\mathrm{c}} \mathrm{dm}^{-3} ; \mathrm{Ca}^{2+}=1,5 \mathrm{cmol}_{\mathrm{c}} \mathrm{dm}^{-3} ; \mathrm{Mg}^{2+}=1,35 \mathrm{cmol}_{\mathrm{c}} \mathrm{dm}^{-3}$; soma de bases de 
$3,02 \mathrm{cmol}_{\mathrm{c}} \mathrm{dm}^{-3} ; \mathrm{CTC}=11,02 \mathrm{cmol}_{\mathrm{c}} \mathrm{dm}^{-3} ; \mathrm{V}=27,40 \% ; \mathrm{m}=21,96 \%$ e matéria orgânica de $34,03 \mathrm{~g} \mathrm{~kg}^{-1}$.

A adubação orgânica foi realizada de acordo com análise química dos estercos, levando-se em consideração apenas o teor de fósforo. Os teores de nutrientes dos estercos foram os seguintes: aves: 7,9 $\mathrm{g} \mathrm{kg}^{-1} \mathrm{de} \mathrm{N}$; $15,8 \mathrm{~g} \mathrm{~kg}^{-1}$ de P e 8,4 $\mathrm{g} \mathrm{kg}^{-1}$ de K. Caprino: $12,8 \mathrm{~g}$ $\mathrm{kg}^{-1}$ de $\mathrm{N} ; 15,2 \mathrm{~g} \mathrm{~kg}^{-1}$ de P e 23,5 $\mathrm{g} \mathrm{kg}^{-1}$ de K. Bovino: 7,4 $\mathrm{g} \mathrm{kg}^{-1}$ de $\mathrm{N}$; 1,5 $\mathrm{g} \mathrm{kg}^{-1}$ de P e 4,6 $\mathrm{g}$ $\mathrm{kg}^{-1}$ de $\mathrm{K}$.

A calagem do solo foi realizada de acordo com a análise química do solo, aplicando-se calcário dolomítico $\left(\mathrm{CaO}\right.$ de 32,3\%; $\mathrm{MgO}$ de $19,3 \% ; \mathrm{SiO}_{2}$ de $\left.0,86 \%\right)$ e gesso agrícola $(\mathrm{CaO}$ de $17,1 \% ; \mathrm{MgO}$ de $1,1 \% ; \mathrm{SiO}_{2}$ de 18,0$)$ na projeção da copa, sendo realizada em todos os tratamentos, inclusive na testemunha.

As variáveis analisadas para os teores foliares foram: nitrogênio, fósforo e potássio (NPK). As folhas foram coletadas nas quatro extremidades da planta, sendo lavadas com água destilada, secas em estufa de circulação de ar a $65^{\circ} \mathrm{C}$ até atingir massa constante. Depois de secas, as folhas foram moídas e encaminhadas para análise, seguindo metodologia de Tedesco (1995) para material vegetal.

Os dados obtidos foram submetidos à análise de variância, utilizando-se até $5 \%$ de significância para o teste F. As médias foram comparadas pelo teste de Tukey a $5 \%$ de probabilidade. Utilizou-se o software $\mathrm{SAS}^{\circledR} 9.3$ (SAS, 2011) para as análises.

\section{Resultados e Discussão}

Com base na Tabela 1, verifica-se que os tratamentos exerceram efeito significativo ( $\mathrm{p} \geq$ $0,01 \%$ ) sobre os teores foliares de NPK das plantas de gravioleira cultivar 'Morada'.

Tabela 1. Resumo da análise de variância para os teores foliares de nitrogênio, fósforo e potássio, em plantas de gravioleira 'Morada' sob a influência da adubação orgânica e mineral.

\section{$\begin{array}{lllll}\text { Fonte de variação } & \text { GL } & \text { Nitrogênio } & \text { Fósforo } & \text { Potássio }\end{array}$}

$\begin{array}{lllll}\text { Blocos } & 3 & 4,5481 & 0,00001909 & 4,7445\end{array}$

$\begin{array}{ccccc}\text { Tratamentos } & 6 & 13,0611^{* *} & 0,00003088^{* *} & 13,4196 * * \\ \text { Resíduo } & 18 & 1,78342 & 0,00000262 & 2,9380 \\ \text { Total } & 27 & 124,1132 & 0,00028965 & 147,6360\end{array}$




$\begin{array}{lllll}\mathrm{C} . \mathrm{V}(\%) & - & 7,0 & 5,9 & 5,4\end{array}$

**: Significativo a $1 \%$ de probabilidade pelo teste $\mathrm{F}$.

Os teores de nitrogênio variaram de 16,1 a 21,4 g/kg (Figura 1). Plantas que receberam adubação mineral, estercos bovino, de aves e de caprino, não apresentaram diferenças significativas. A maior média foi observada no tratamento com esterco caprino $(21,4 \mathrm{~g} / \mathrm{kg})$.

Os menores teores foram observados na testemunha com $16,1 \mathrm{~g} / \mathrm{kg}$, motivado pela ausência de adubação. Todos os teores de $\mathrm{N}$ obtidos estão na faixa de suficiência proposta por Avilán (1975) e Batista et al. (2003) para a gravioleira, os quais mencionam como adequados teores entre 14,7 e 17,6 g/ $\mathrm{kg}$ e como deficientes teores entre 8,8 a 11,0 $\mathrm{g} / \mathrm{kg}$. Para Andrade (2004) os teores de $\mathrm{N}$ devem variar de 17 a $28 \mathrm{~g} / \mathrm{kg}$. No entanto, Lima (2012) avaliando a composição mineral da gravioleira submetida à adubação nitrogenada, obteve valores semelhantes aos determinados nesse estudo $(21,6 \mathrm{~g} / \mathrm{kg})$.

Figura 1. Teores foliares de nitrogênio, em plantas de gravioleira, sob influência da adubação orgânica e mineral.

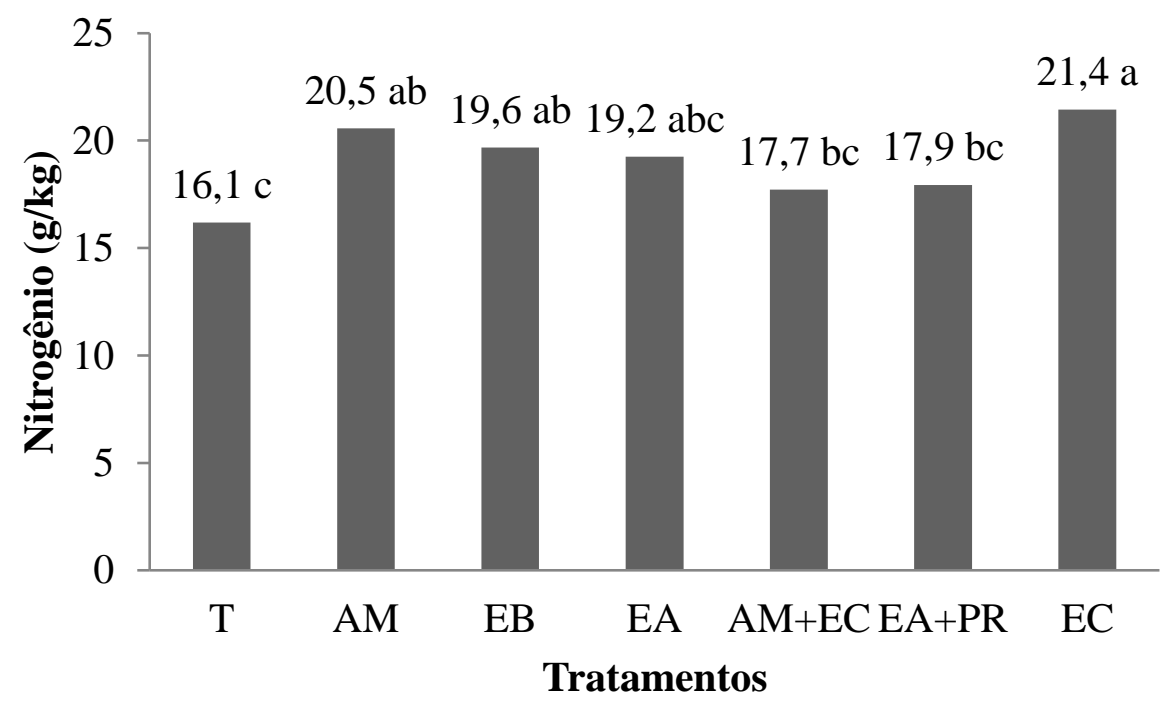

Médias seguidas pela mesma letra não diferem entre si pelo teste de Tukey a 5\% de probabilidade. $\mathrm{T}=$ testemunha; $\mathrm{AM}=$ adubação mineral; $\mathrm{EB}=$ esterco bovino; $\mathrm{EA}=$ esterco de aves; $\mathrm{AM}+\mathrm{EC}=$ adubação mineral mais esterco caprino; $\mathrm{EA}+\mathrm{PR}=$ esterco de aves mais pó de rocha; $\mathrm{EC}=$ esterco caprino.

O tratamento com esterco bovino apresentou os maiores teores de fósforo $(0,31 \mathrm{~g} / \mathrm{kg})$ foliar dentre os demais tratamentos avaliados (Figura 2). Os menores valores foram 
observados na testemunha e no esterco caprino, ambos com 0,23 g/kg. Estes teores estão abaixo dos valores observados por Frazão et al. (2002), que foi de 0,47 a 0,92 g/kg de P. Viégas e Frazão (2004) determinam os valores de 0,8 a 1,0 g/kg de P, como adequados para nutrição de fósforo foliar. No entanto, Silva et al. (1986) sugerem como adequados para a gravioleira aos teores de 1,4 a 1,5 g/kg. Na gravioleira, Avilán (1975) e Silva et al. (1986) observaram, com a omissão de fósforo, redução no porte da planta, em relação ao tratamento completo, com sintomas de deficiência inicialmente nas folhas inferiores, atingindo, em seguida, as folhas medianas e superiores.

Os menores valores para $\mathrm{P}$, podem estar relacionado à interação inversa existente entre as concentrações de N e P descrita por Smith (1966), Reese e Koo (1975). De acordo com esses pesquisadores, plantas com teores baixos de $\mathrm{N}$ poderão apresentar altos teores de $\mathrm{P}$; porém, quando há altos teores de $\mathrm{N}$ geralmente ocorrem baixos teores de $\mathrm{P}$, sendo difícil encontrar folhas com excesso de $\mathrm{N}$ e $\mathrm{P}$ ao mesmo tempo (SILVA, 2012).

Figura 2. Teores foliares de fósforo, em plantas de gravioleira, sob influência da adubação orgânica e mineral.

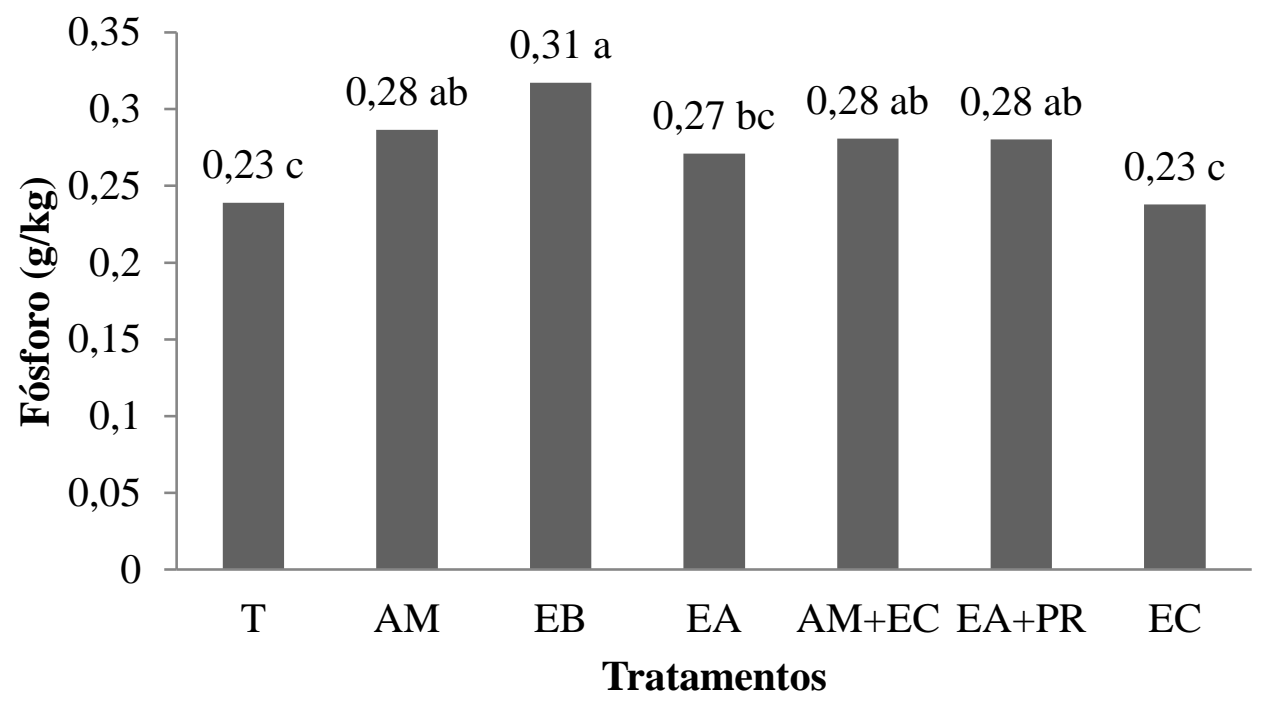

Médias seguidas pela mesma letra não diferem entre si pelo teste de Tukey a 5\% de probabilidade.

$\mathrm{T}=$ testemunha; $\mathrm{AM}=$ adubação mineral $\mathrm{EB}=$ esterco bovino; $\mathrm{EA}=$ esterco de aves; $\mathrm{AM}+\mathrm{EC}=$ adubação mineral mais esterco caprino; $\mathrm{EA}+\mathrm{PR}=$ esterco de aves mais pó de rocha; $\mathrm{EC}=$ esterco caprino.

O maior teor de potássio foliar foi observado no tratamento com esterco bovino (34 $\mathrm{g} / \mathrm{kg}$ ) (Figura 3). Não houve efeito significativo entre os tratamentos AM, EA, AM+EC e $\mathrm{AM}+\mathrm{PR}$. Os menores teores foram observados nos tratamentos $\mathrm{T}(28,5 \mathrm{~g} / \mathrm{kg})$ e EC $(29,4$ 
g/kg). Avilán (1975) e Oliveira (2004) consideram adequado teor de $\mathrm{K}^{+}$foliar quando acima de $26 \mathrm{~g} / \mathrm{kg}$, e como insuficientes abaixo de 12,6 g/kg. Haridasan (1987) considera suficiente quando acima de 6,4 g/kg, e para Andrade (2004) este teor foliar deve estar entre 18 e $26 \mathrm{~g} / \mathrm{kg}$ de $\mathrm{K}^{+}$. Assim, nenhum dos teores obtidos estão abaixo da faixa de suficiência determinada por estes pesquisadores.

Figura 3. Teores foliares de potássio, em plantas de gravioleira, sob influência da adubação orgânica e mineral.

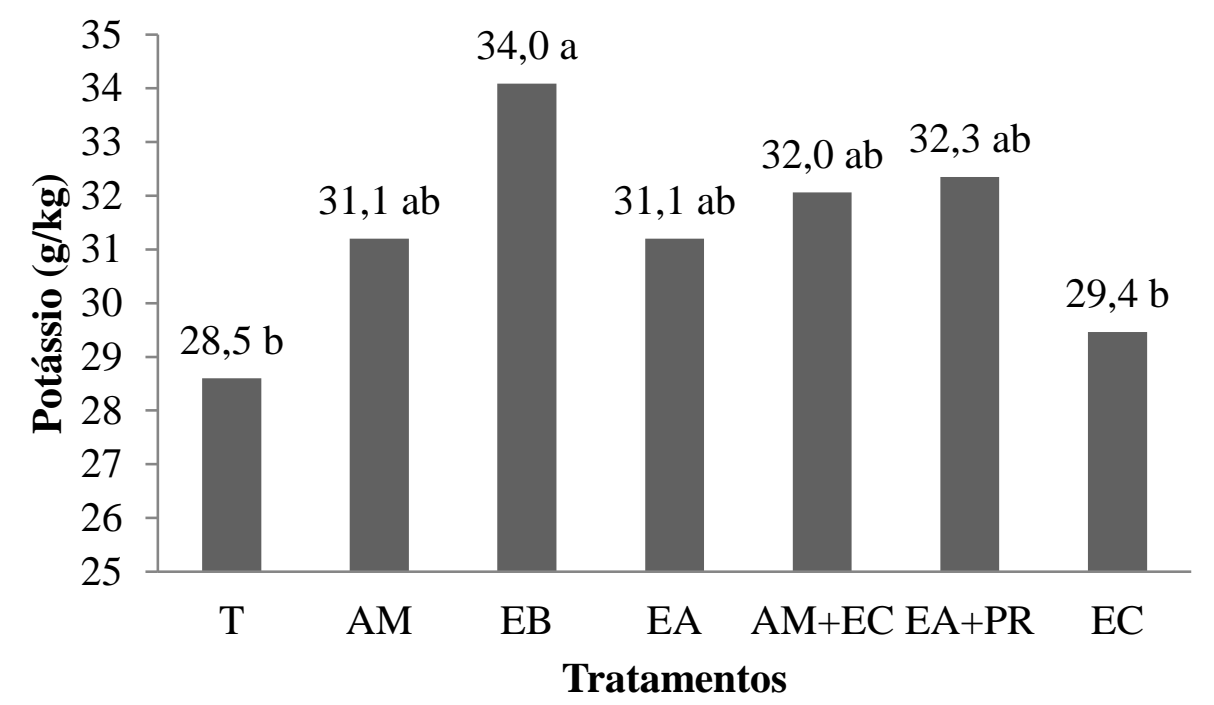

Médias seguidas pela mesma letra não diferem entre si pelo teste de Tukey a 5\% de probabilidade. $\mathrm{T}=$ testemunha; $\mathrm{AM}=$ adubação mineral; $\mathrm{EB}=$ esterco bovino; $\mathrm{EA}=$ esterco de aves; $\mathrm{AM}+\mathrm{EC}=$ adubação mineral mais esterco caprino; $\mathrm{EA}+\mathrm{PR}=$ esterco de aves mais pó de rocha; $\mathrm{EC}=$ esterco caprino.

\section{Considerações finais}

A adubação orgânica com esterco bovino proporcionou maiores teores foliar de fósforo e potássio;

O esterco caprino, seguido da adubação com NPK e esterco bovino proporcionaram maiores teores foliar de nitrogênio. 


\section{Referências}

ABREU, N. A. A. et al. Crescimento de mudas de pitangueira (Eugenia uniflora L.) em substratos com utilização de superfosfato simples. Ciência e Agrotecnologia, Lavras, v. 29, n. 6, p. 1117-1124, nov./dez. 2005.

ANDRADE, L. R. M. Corretivos e fertilizantes para culturas perenes e semiperenes. In: SOUSA, D. M. G. LOBATO, E. (Ed.). Cerrado: correção do solo e adubação. Brasília: Embrapa Informação Tecnológica, 2004. p. 317-366.

AVILÁN, L. R. Efecto de la omisión de los macronutrientes en el desarrollo y composición química da la guanábana (Annona muricata L.) cultivada en soluciones nutritivas.

Agronomia Tropical, Maracay, v.25, n.1, p.73 - 79, 1975.

BATISTA, M. M. F.; VIÉGAS, I. J. M.; FRAZÃO, D. A. C. F.; THOMAZ, M. A. A.; SILVA, R. C. L. Efeito da omissão de macronutrientes no crescimento, nos sintomas de deficiências nutricionais e na composição mineral em gravioleiras (Annona muricata L.).

Revista Brasileira de Fruticultura, v.25, p.315-318, 2003.

BRASIL, Ministério da Agricultura. Levantamento exploratório-reconhecimento de solos do estado da Paraíba. Rio de Janeiro: MA/SUDENE, 669 p. (Boletim Técnico, 15). 1972.

CAVALCANTI, F. J. A. (Coord.). Recomendações de adubação para o estado do

Pernambuco ( $2^{\mathbf{a}}$ aproximação). 3. ed. Recife: Instituto Agronômico do Pernambuco-IPA, 2008. 212 p.

FRAZÃO, D. A. C.; VIÉGAS, I. J. M.; BATISTA, M. M. F.; CRUZ, E. S.; SILVA, J. F. Teores de N, P, K, Ca, Mg e S, em gravioleiras cultivadas em solução nutritiva com omissão de macronutrientes. In: Congresso Brasileiro de Fruticultura, 17, 2002, Belém. Anais... Belém: S.B.F, 2002. CD Rom.

FREITAS, A. L. G. E.; VILASBOAS, F. S.; PIRES, M. M.; SÃO JOSÉ, A. R. Caracterização da produção e do mercado da graviola (Annona muricata L.) no estado da Bahia.

Informações Econômicas, SP, v. 43, n. 3, maio/jun. 2013.

GOMES JÚNIOR, G. A.; PEREIRA, R. A.; SACRAMENTO, C. K.; SOUZA JÚNIOR, J. O. EXTRAÇÃO E EXPORTAÇÃO DE NUTRIENTES EM FRUTOS DE GRAVIOLEIRA.

Ciência Agrícola, Rio Largo, v. 16, n. 2, p. 80-84, 2018.

GONDIM, A. W. A. Geoeconomia e Agricultura do Brejo Paraibano: análise e avaliação. João Pessoa: Imprensa Universitária, 82p. 1999.

HARIDASAN, M. 1987. Distribution and mineral nutrition of aluminium-accumulating species in different plant communities of the cerrado region of Central Brazil. In La capacidad bioproductiva de sabanas. Instituto Venezolano de Investigaciones Cientificas, Caracas, p.309-348.

HOLANDA FILHO, R. S. F.; SOUSA, V. F. de; AZEVEDO, B. M.; ALCANTARA, R. M. C. M.; RIBEIRO, V. Q.; ELOI, W. M. Efeitos da fertirrigação de $\mathrm{N}$ e $\mathrm{K}_{2} \mathrm{O}$ na absorção de 
macronutrientes pela gravioleira. Revista Brasileira de Engenharia Agrícola e Ambiental. v. 10, n.1, p. 43-49, 2006.

LIMA, J. S. Crescimento, eficiência quântica potencial do fotossistema II e composição mineral da gravioleira 'Morada' sob adubação nitrogenada. Dissertação (Mestrado em Agronomia), Centro de Ciências Agrárias, Universidade Federal da Paraíba, Areia, 2012.

MALAVOLTA, E. Importância da adubação na qualidade dos produtos/função dos nutrientes na planta. In: SIMPÓSIO SOBRE ADUBAÇÃO E QUALIDADE DOS PRODUTOS AGRÍCOLAS, 1, 1989, Ilha Solteira, SP. Anais... São Paulo: Ícone, 1994. p.19-51.

NATALE, W.; COUTINHO, E. L. M.; BOARETTO, A. E.; CORTEZ G. E. P.; FESTUCCIA, A. J. Extração de nutrientes por frutos de goiabeira (Psidium guajava L.). Científica, São Paulo, v.22, n.2, p.249-253. 1994.

OLIVEIRA, S. A. de. Análise foliar. In: SOUSA, D. M. G. de; LOBATO, E. (Ed.). Cerrado: correção do solo e adubação. Brasília: Embrapa Informação Tecnologia, 2004. p.245-282.

REESE, R.L.; KOO, R.C.J. Effects of N and K fertilization on leaf analysis, tree size and yield of three major Florida orange cultivars. Journal of the American Society for Horticultural Science, Winter Haven, v.100, p.195-198, 1975.

SACRAMENTO, C. K.; FARIA, J. C.; CRUZ, F.; L. Caracterização física e química de frutos de três tipos de gravioleira (Annona muricata L.). Revista Brasileira de Fruticultura, ago. 2003, vol.25, no.2, p.329-331. ISSN 0100-2945.

SAMARÃO, S. S.; RODRIGUES, L. A.; MARTINS, M. A.; MANHÃES, T. N.; ALVIM, L. A. M. Desempenho de mudas de gravioleira inoculadas com fungos micorrízicos arbusculares em solo não-esterilizado, com diferentes doses de fósforo. Acta Scientiarum. Agronomy, Maringá, v. 33, n. 1, p. 81-88, 2011.

SÃO JOSÉ, A. R. Cultivo e mercado da graviola. Fortaleza: Instituto Frutal, 2003. 36 p.

SÃO JOSÉ, A. R.; PRADO, N. B.; BONFIM, M. P.; REBOUÇAS, T. N. H.; MENDES, H. T. A. Marcha de absorção de nutrientes em anonáceas. Revista Brasileira de Fruticultura. v. 36, edição especial, e., p. 176-183, fev 2014.

SILVA, A. Q.; SILVA, H.; ROQUE M. L.; MALAVOLTA, E. Nutrição mineral da graviola (Annona muricata L.), sintomas de carências nutricionais. In: CONGRESSO BRASILEIRO DE FRUTICULTURA, 8. 1986, Brasília. Anais... Brasília: SBF/EMBRAPA, 1986. v. 2, p. 297-301.

SILVA, A.V.C.; MUNIZ, E.N.; RANGEL, J.H.A.; YAGUIU, P.; SOUZA, J.P.B.; CARNELOSSI, M.A.G. Quality of custard apple (Annona squamosal L.) in relation to packaging and storage period. Acta Horticulturae, The Hague, v.934, p. 707-712, 2012.

SOARES, I.; LIMA, C. S.; CRISÓSTOMO, A. L.; Crescimento e composição mineral de mudas de gravioleira em resposta a doses de fósforo. Rev. Ciên. Agron., Fortaleza, v.38, n.4, p.343-349, Out.- Dez., 2007. 
SOUZA, C. A. S.; CORRÊA, F. L. O.; MENDONÇA, V.; CARVALHO, J. G. CRESCIMENTO DE MUDAS DE GRAVIOLEIRA (Anonna muricata L.) EM SUBSTRATO COM SUPERFOSFATO SIMPLES E VERMICOMPOSTO. Rev. Bras. Frutic., Jaboticabal - SP, v. 25, n. 3, p. 453-456, dezembro 2003.

SMITH, P. F. Citrus nutrition. In: CHILDERS, N.F. (Ed.). Nutrition of fruit crops: temperate to tropical fruit. New Brunswick: Rutgers the State of Universit, 1966. p.174-207.

STATISTICAL ANALYSIS SYSTEM INSTITUTE, SAS - Institute SAS/STAT software 2011, CD-ROOM.

TEDESCO, M.J.; GIANELLO, C.; BISSANI, C.A.; BOHNEN, H. \& VOLKWEISS, S.J. Análise de solos, plantas e outros materiais. Porto Alegre, Universidade Federal do Rio Grande do Sul, 1995. 174p. (Boletim Técnico, 5).

TELES, A. C. M.; PINTO, E. G.; SANTOS, J. R.; OLIVEIRA, C. F. D.; SOARES, D. S. B. Desenvolvimento e caracterização físico-química de geleia comum e extra de graviola com pimenta. Revista de Agricultura Neotropical, Cassilândia-MS, v. 4, n. 1, p. 72-77, jan./mar. 2017.

VIÉGAS, I. J. M.; FRAZÃO, D. A. C. Graviola: nutrição, calagem e adubação. Belém: EMBRAPA Amazônia Oriental, 2004. 6p. (Circular Técnica, 36). 